\title{
Insuficiência cardíaca no Brasil: enfoquenas internações hospitalares no período de 2010 a 2019
}

\author{
Heart failure in Brazil: focus on hospital admissions from 2010 to 2019
}

Rayane de Oliveira Silva Santos ${ }^{1 *}$, Sara Cristine Marques dos Santos ${ }^{1}$, Gabriela Albuquerque dos Santos ${ }^{2}$, Mariana Luíza Ferreira de Azevedo ${ }^{1}$, Thaísa Pimenta Ferreira de Oliveira ${ }^{1}$, Ivana Picone Borges de Aragão ${ }^{3}$

Como citar esse artigo. Santos,

R.O.S; dos Santos, S.C.M; dos

Santos, G.A; de Azevedo, M.L.F; de Oliveira, T.P.F; de Aragão, I.P.B Insuficiência cardíaca no Brasil: enfoque nas internações hospitalares no período de 2010 a 2019. Revista de Saúde. 2021 Abr./Jul.; 12 (2): 37-40.

\section{Resumo}

Anualmente, cerca de 2 milhões de casos novos de insuficiência cardíaca são diagnosticados no mundo e, no Brasil, a doença constitui a primeira causa de internação hospitalar em pacientes com idade superior a 60 anos, com chance de readmissão de até $50 \%$. Diante disso, o objetivo do presente estudo é analisar as internações hospitalares, valor gasto e o tempo médio de permanência por insuficiência cardíaca, entre 2010 e 2019, no Brasil. Foi realizado estudo ecológico, observacional e retrospectivo, através da análise de dados do Departamento de Informática do Sistema Único de Saúde (DATASUS), pelo Sistema de Informações Hospitalares (SIH) e Sistema de Morbidade Hospitalar (SIM), acerca do CID10 I50.0, I50.1 e I50.9. No período sinalizado, as internações por insuficiência cardíaca corresponderam a $2 \%$ do total de internações, das quais $94,4 \%$ foram de caráter urgente; 70,3\% dos casos ocorreram naqueles acima de 60 a 69 anos; a mortalidade intra-hospitalar proporcional foi de 10,1\%. O presente estudo demonstrou que o número de internações por insuficiência cardíaca diminuiu entre 2010 e 2019, o que se deve ao surgimento de novos tratamentos da doença, todavia, a mortalidade intra-hospitalar proporcional ainda é alta e foi maior nos dois últimos anos analisados. A internação por urgência é mais comum, se relacionando ao agravamento súbito dos sintomas, configurando insuficiência cardíaca aguda. Além disso, os idosos são mais internados pelo fato de serem mais acometidos pela doença e comorbidades. Com isso, concluiu-se que políticas públicas de promoção em saúde e prevenção primária são necessárias, para reduzir a incidência da doença e, consequentemente, a necessidade de internação.

Palavras-chave: Insuficiência cardíaca; Insuficiência cardíaca diastólica; Insuficiência cardíaca sistólica.

\begin{abstract}
Annually, about 2 million new cases of heart failure are diagnosed in the world and, in Brazil, the disease is the first cause of hospitalization in patients over 60 years, with a chance of readmission of up to $50 \%$. Therefore, the objective of the present study is to analyze hospital admissions caused by heart failure, between 2010 and 2019, in Brazil. An ecological, observational and retrospective study was carried out, through data analysis from the Informatics Department of the Unified Health System (DATASUS), through the Hospital Information System (SIH) and Hospital Morbidity System (SIM), about the ICD-10 I50.0, I50.1 and I50.9. In the flagged period, hospitalizations for heart failure corresponded to $2 \%$ of the total hospitalizations, of which $94.4 \%$ were of an urgent nature; $70.3 \%$ of cases occurred in those over 60 to 69 years old; proportional in-hospital mortality was $10.1 \%$. Our study showed that the number of hospitalizations for heart failure decreased between 2010 and 2019, because of the emergence of new treatments for the disease, however, the proportional in-hospital mortality is still high and was higher in the last two years analyzed. Urgent hospitalization is more common, as it relates to the sudden worsening of the symptoms, constituting acute heart failure. In addition, the elderly was more hospitalized because they are more affected by the disease and comorbidities. Thus, it was concluded that public policies for health promotion and primary prevention are necessary to reduce the incidence of the disease and, consequently, the need for hospitalization.
\end{abstract}

Keywords: Heart failure; Heart failure, diastolic; Heart failure, systolic.

\section{Introdução}

A insuficiência cardíaca (IC) corresponde ao conjunto de sinais e sintomas consequentes à incapacidade do coração em ejetar o sangue de forma a alcançar as necessidades metabólicas do corpo $^{1,2}$. Essa síndrome pode ser causada por alterações na estrutura ou no funcionamento cardíaco e caracterizase por manifestações,no repouso ou durante o esforço, que resultam da redução do débito cardíaco e/ou das elevadas pressões de enchimento ${ }^{1}$.

A IC pode ser classificada de acordo com a gravidade dos sintomas, o tempo de evolução da doença e a fração de ejeção do ventrículo esquerdo. O último parâmetro é responsável por dividir a doença em IC com fração de ejeção preservada (ICFEP) e IC com fração de ejeção reduzida (ICFER) $)^{1,3}$. Atualmente, a doença é responsável por um prognóstico de alta morbimortalidade e apesar de existirem

2- Graduada em Educação Física pela Universidade Castelo Branco, Rio de Janeiro, Brasil.

3- Docente do Curso de Medicina da Universidade de Vassouras, Rio de Janeiro, Brasil. 
tratamentos eficazes para ICFER, ainda há carência no desenvolvimento de terapias para ICFEP ${ }^{4,5}$.

Anualmente, cerca de 2 milhões de casos novos de IC são diagnosticados no mundo ${ }^{6} \mathrm{e}$ acreditase que, em países desenvolvidos, a doença acomete entre 5 a 10 pessoas por 1.000 habitantes, determinando custos diretos com a doença de aproximadamente $2 \%$ do orçamento total para saúde 7 . No Brasil, a doença constitui a primeira causa de internação hospitalar em pacientes com idade superior a 60 anos e a chance de readmissão pode chegar a $50 \%$ após a alta ${ }^{6,2}$. Apesar dessa faixa etária ser mais prevalente, é sabido que em países subdesenvolvidos há subnotificação de IC pediátrica, principalmente naquelas portadoras de cardiopatia congênita ${ }^{8} \mathrm{e}$ estima-se que, anualmente nos EUA, cerca de 11.000 a 14.000 crianças são internadas por IC anualmente ${ }^{2}$.

Diante disso, o objetivo do presente estudo é analisar as internações hospitalares, valor gasto e o tempo médio de permanência por IC, entre 2010 e 2019 , no Brasil.

\section{Metodologia}

Foi realizado estudo ecológico, observacional e retrospectivo, através da análise de dados obtidos no banco de dados do Departamento de Informática do Sistema Único de Saúde (DATASUS) pelo Sistema de Informações Hospitalares (SIH) e pelo Sistema de Morbidade Hospitalar (SIM), acerca do CID-10 I50.0, I50.1 e I50.9.

Foram analisados o número total de internações hospitalares, os quais foram relacionados ao sexo (masculino e feminino) e faixa etária ( 1 a 4 anos, 5 a 9 anos, 10 a 14 anos, 15 a 19 anos, 20 a 29 anos, 30 a 39 anos, 40 a 49 anos, 50 a 59 anos, 60 a 69 anos, 70 a 79 anos e 80 anos ou mais); o caráter da internação (urgência ou eletivo), número de óbitos e mortalidade proporcional por IC, no período de janeiro de 2010 a dezembro de 2019.

Os dados coletados foram analisados por meio do programa Microsoft Excel $\AA$, usando a porcentagem e os números absolutos.

Não houve submissão ao Conselho de Ética em Pesquisa (CEP) devido ao DATASUS ser um banco de dados de domínio público, sem identificação dos pacientes cadastrados.

\section{Resultados}

No período de janeiro de 2010 a dezembro de 2019, foram notificadas 2.321.102 internações por IC no Brasil, o que corresponde a 2,0\% do total de internações hospitalares (114.392.584). Dessas, 2.203.055 (94,9\%) foram de caráter urgente e 118.058 (5,1\%) eletivo. O ano com maior número de internações foi 2010 , com

Tabela 1. Mortalidade intra-hospitalar proporcional por insuficiência cardíaca, número absoluto de internações e óbitos, estratificadas por ano, no Brasil.

\begin{tabular}{cccc}
\hline Ano & Internações & Óbitos & $\begin{array}{c}\text { Mortalidade } \\
\text { proporcional (\%) }\end{array}$ \\
\hline $\mathbf{2 0 1 0}$ & 272.350 & 23.918 & 8.8 \\
\hline $\mathbf{2 0 1 1}$ & 266.371 & 24.589 & 9.2 \\
\hline $\mathbf{2 0 1 2}$ & 246.900 & 23.122 & 9.4 \\
\hline $\mathbf{2 0 1 3}$ & 242.438 & 23.060 & 9.5 \\
\hline $\mathbf{2 0 1 4}$ & 227.346 & 22.083 & 9.7 \\
\hline $\mathbf{2 0 1 5}$ & 221.717 & 22.976 & 10.4 \\
\hline $\mathbf{2 0 1 6}$ & 218.558 & 23.771 & 10.9 \\
\hline $\mathbf{2 0 1 7}$ & 212.294 & 22.817 & 11.1 \\
\hline $\mathbf{2 0 1 8}$ & 204.836 & 22.765 & 11.2 \\
\hline $\mathbf{2 0 1 9}$ & 191.697 & 21.541 & \\
\hline
\end{tabular}

Fonte: Autores (2021)

Tabela 2. Mortalidade intra-hospitalar proporcional por IC, número absoluto de internações e óbitos, estratificados de acordo com a faixa etária, no período de 2010 a 2019, no Brasil..

\begin{tabular}{|c|c|c|c|}
\hline Faixa etária & Internações & Óbitos & Mortalidade proporcional (\%) \\
\hline Menor 1 ano & 13.374 & 1.414 & 10,6 \\
\hline 1 a 4 anos & 7.760 & 354 & 4,6 \\
\hline 5 a 9 anos & 4.544 & 182 & 4,0 \\
\hline 10 a 14 anos & 5.099 & 320 & 6,3 \\
\hline 15 a 19 anos & 7.765 & 593 & 7,6 \\
\hline 20 a 29 anos & 26.198 & 1.998 & 7,6 \\
\hline 30 a 39 anos & 63.471 & 4.389 & 6,9 \\
\hline 40 a 49 anos & 159.324 & 10.501 & 6,6 \\
\hline 50 a 59 anos & 355.933 & 25.441 & 7,1 \\
\hline 60 a 69 anos & 536.127 & 46.039 & 8,6 \\
\hline 70 a 79 anos & 600.400 & 63.423 & 10,6 \\
\hline 80 anos ou mais & 494.506 & 75.790 & 10,3 \\
\hline
\end{tabular}

Fonte: Autores (2021)

272.350 admissões e o ano de 2019 o menor, com 191.697(Tabela 1).

Do total de internações, $1.167 .672(50,3 \%)$ eram indivíduos do sexo masculino e $1.106 .759(47,7 \%)$ correspondiam a pacientes do sexo feminino. 
No que tange a faixa etária dos pacientes internados por IC, foram registrados $600.381(25,9 \%)$ casos entre indivíduos 70 e 79 anos, 536.112 (23,1\%) entre os 60 e 69 anos e 494.483 (21,3\%) naqueles acima de 80 anos, que somados, representam um total de $1.630 .976(70,3 \%)$ casos. Analisando a idade correspondente a pacientes pediátricos, o menor número de casos ocorreu na faixa etária entre 5 a 9 anos, com $4.544(0,2 \%)$ casos e a maior prevalência foi naqueles com idade inferior a 1 ano, totalizando $13.374(0,6 \%)$ casos(Tabela 2).

Levando em consideração o total de internações hospitalares do período, os gastos foram de R\$ 133.004.158.877,10, sendo R \$3.042.891.611,81 (2,3\%) utilizados apenas em internamentos causados por IC.

Em relação ao tempo de permanência hospitalar no Brasil, as internações por IC, independentemente do caráter eletivo ou de urgência, corresponderam a uma média de internação de 7,1 dias.

No Brasil, levando em consideração as internações hospitalares por IC, o total de óbitos registrados no período foi de 230.440 , correspondendo a uma mortalidade proporcional de $10,1 \%$. Apesar do maior número absoluto por mortes ter ocorrido em 2011 (24.589), a maior mortalidade proporcional ocorreu em 2019 , com $11,2 \%$ e a menor em 2010 , com $8,8 \%$. (Tabela 1).

\section{Discussão}

A IC apresenta diferentes formas de classificação funcional, como a elaborada pela New York Heart Association (NYHA), que leva em consideração a gravidade dos sintomas apresentados e a determinada pelo American College of Cardiology/American Heart Association (ACC/AHA), que classifica a doença de acordo com seu tempo de evolução. A segunda classificação citada indica necessidade de terapias particularizadas em pacientes com estágios avançados ${ }^{1}$.

O presente estudo demonstrou que a IC ainda é responsável por uma pequena parcela das internações hospitalares, com porcentagem similar àquela encontrada em estudo de Ziaeian e Fonarow, nos Estados Unidos da América (EUA), em 2016 (1-2\%) Acredita-se que a baixa incidência de admissões hospitalares pela doença ocorre devido ao avanço farmacológico em seu tratamento, com introdução de drogas responsáveis pela melhora da classe funcional e redução das hospitalizações, como os inibidores da enzima conversora de angiotensina, betabloqueadores, inibidores de neprisilina e inibidores do co-transportador de sódio-glicose-2 (SGLT2) ${ }^{9-17}$. Todavia, apesar de ter sido demonstrada queda no número de internamentos motivados pela doença, ao longo dos 10 anos analisados no Brasil, no país norte-americano foi constatado aumento do número de internações, entre 1979 e $2004^{8}$.

Ademais, foi observado que a maior parte das internações ocorrem em caráter de urgência. Tal fato pode estar relacionado ao agravamento súbito dos sintomas, configurando uma síndrome de IC aguda, com piora progressiva da função cardíaca e qualidade de vida ${ }^{18}$.

Em relação ao sexo mais internado, foi encontrada proporção similar entre homens e mulheres, diferindo da informação encontrada no estudo Breathe, realizado em 2015, em 51 hospitais brasileiros, da rede pública e privada, em que $60 \%$ dos pacientes eram do sexo feminino ${ }^{19}$. Tal diferença pode ter ocorrido pois o período analisado neste estudo foi maior, além de abranger todos os hospitais que fornecem as informações ao DATASUS.

A incidência da IC tem aumentado em razão do envelhecimento da população e a existência de comorbidades cardíacas e não cardíacas ${ }^{18}$, como aumento da ocorrência de diabetes mellitus, hipertensão e da sobrevivência após o infarto do miocárdio ${ }^{20}$, elevando o risco de hospitalização e reinternação ${ }^{18}$, o que corrobora com a faixa etária prevalente encontrada neste estudo, com maior número de internações a partir dos 60 anos. Além disso, a faixa etária também foi similar àquela encontrada por Poffo et al. ${ }^{6}$, que demonstraram que a média de idade em indivíduos internados por IC, em hospital terciário de Santa Catarina, entre 2010 e 2014, era de 66,5 anos,bem como nos EUA, em que a média foi de 65 anos ou mais 8 .

Apesar de ser doença majoritariamente encontrada em adultos, a IC também pode estar presente em crianças, decorrente em sua maioria de distúrbios circulatórios de sobrecarga de volume e obstrução de fluxo ${ }^{21}$, sendo a cardiopatia congênita e cardiomiopatia primária as causas mais comuns ${ }^{2}$. Analisando a faixa etária pediátrica, este estudo demonstrou que o número de hospitalizações e a mortalidade proporcional foram maiores naqueles com idade inferior a 1 ano, o que pode ser explicado pelo fato de que aproximadamente $60 \%$ dos casos de IC em crianças ocorrem antes e durante o primeiro ano de vida e aquelas que necessitam de hospitalização apresentam mortalida de 20 vezes superior em relação ao restante da população pediátrica ${ }^{2}$.

Além disso, o presente estudo evidenciou que milhões de reais já foram gastos pelo Sistema Único de Saúde com as internações hospitalares por IC no Brasil. Para que haja redução dos gastos públicos, é necessário que a prevenção primária seja estimulada, como tratamento correto de hipertensos, políticas de conscientização sobre tabagismo e mudança de estilo de vida. Com isso, a redução da incidência da doença se torna possível e, por consequência, o número de admissões hospitalares ${ }^{8}$. 
A mortalidade em internações por IC é proporcional à quantidade de dias que o indivíduo permanece internado e foi demonstrado que a morte súbita (MS) pode ocorrer em até 14 dias de internação ${ }^{6}$. Este estudo demonstrou uma média razoável de dias de hospitalização no Brasil,se mostrando inferior àquela encontrada por Zhang et al. ${ }^{22}$, em estudo realizado na China, entre janeiro de 2012 a setembro de 2015, que demonstraram média de permanência de 10 dias.

Em relação à mortalidade hospitalar proporcional por IC, o estudo demonstrou uma taxa elevada, principalmente nos dois últimos anos de estudo e proporção similar foi encontrada por Poffo e colaboradores $(11,2 \%)^{6}$. Entretanto, Zhang et al. ${ }^{22}$, demonstraram que a mortalidade intra-hospitalar encontrada foi de $4,1 \%$, valor muito inferior àquele encontrado em território brasileiro. Além disso, estudo realizado em 2012, no Sistema Nacional de Saúde Espanhol, mostrou que as taxas de mortalidade variam de acordo com a quantidade de internações do paciente, demonstrando $9,2 \%$ de mortalidade no primeiro evento, atingindo $14,5 \%$ em um ano de seguimento ${ }^{23}$.

\section{Conclusão}

Destarte, concluiu-se que apesar de as internações hospitalares por IC corresponderem a uma baixa porcentagem do total de admissões, a sua mortalidade intra-hospitalar ainda se mantém elevada. Além disso, embora a faixa etária dos idosos seja a mais acometida, ainda é possível notar internamentos na população pediátrica, principalmente naquela com idade inferior a um ano.

Diante disso, é necessário que sejam realizadas políticas de promoção em saúde e prevenção primária, principalmente na população adulta que cursa com mais comorbidades que predispõe à evolução para IC e, consequentemente, maior risco de descompensação da doença e internação.

Ademais, embora a média de permanência hospitalar seja razoável, é imprescindível que haja redução no tempo de internação e aprimoramento das técnicas intra-hospitalares, para que haja redução da mortalidade intra-hospitalar.

O presente estudo apresenta limitações, uma vez que utilizou informações de bancos de dados, que estão sujeitos a revisão e a subnotificação.

\section{Referências}

1. Rohde LEP, Montera MW, Bocchi EA, Clausell NO, Albuquerque DC, Rassi S, et al. Diretriz brasileira de insuficiência cardíaca crônica e aguda. Arq Bras Cardiol 2018;111(3):436-539.

2. Hinton RB, Ware SM. Heart Failure in Pediatric Pacients With Congenital Heart Disease. Circ Res 2017; 120(6):978-994.

3. Ponikowski P, Voors AA, Anker SD, Bueno H, Cleland JGF, Coats
AJS, et al. ESC Guidelines for the diagnosis and treatment of acute and chronic heart failure. European Heart Journal2016; 37:2129-2200.

4. Davison B, Cotter G. Why is heart failure so important in the $21 \mathrm{st}$ century? Eur J Heart Fail 2015;17(2):122-124.

5. Van Der Meer P, Gaggin HK, Dec GW. ACC/AHA Versus ESC Guidelines on Heart Failure. JAm Col of Cardiol. 2019; 73 (21):2756-2768

6. Poffo MR, Assis VA, Fracasso M, Miguel O, Filho L, Matos S, et al. Perfil dos Pacientes Internados por Insuficiência Cardíaca em Hospital Terciário. Int J Cardiovasc Sci2017;30(3):189-198.

7. Fonseca C, Brás D, Araújo I, Ceia F. Insuficiência cardíaca em números: estimativas para o século XXI em Portugal. Ver PortCardiol 2018; 37(2):97-104.

8. Ziaeian B, Fonarow GC. Epidemiology and aetiology of heart failure Nat Rev Cardiol 2016;13:368-378.

9. The SOLVD Investigators. Studies of Left Ventricular Dysfunction (SOLVD)-Rationale, design and methods: Two trials that evaluate the effect of enalapril in patients with reduced ejection fraction. Am J Cardiol 1990; $66(3): 315-322$

10. Swedberg K, Idanpaan-Heikkila U, Remes J. Effects of enalapril on mortality in severe congestive heart failure. Results of the Cooperative North Scandinavian Enalapril Survival Study (CONSENSUS). N Engl J Med 1987;316(23):1429-1435.

11. Dargie HJ, Lechat P. The Cardiac Insufficiency Bisoprolol Study II (CIBIS-II): A randomised trial. Lancet1999;353(9146):9-13.

12. Hjalmarson A, Goldstein S, Fagerberg B, Wedel H, Waagstein $\mathrm{F}$, Kjekshus J, et al. Effect of metoprolol $\mathrm{CR} / \mathrm{XL}$ in chronic heart failure: Metoprolol CR/XL Randomised Intervention Trial in Congestive Heart Failure (MERIT-HF). Lancet 1999;353(9169):2001-2007.

13. Dargie HJ. Effect of carvedilol on outcome after myocardial infarction in patients with left-ventricular dysfunction: The CAPRICORN randomised trial. Lancet2001;357(9266):1385-1390.

14. Eichhorn EJ, Bristow MR. The Carvedilol Prospective Randomized Cumulative Survival (COPERNICUS) trial. Curr Control Trials Cardiovasc Med2001; 2(1):20-23.

15. Yan AT, Yan RT, Liu PP. Narrative Review: Pharmacotherapy for Chronic Heart Failure: Evidence from Recent Clinical Trials. Ann Intern Med2005 18;142(2):132-145.

16. Cuthbert JJ, Pellicori P, Clark AL. Cardiovascular Outcomes with Sacubitril-Valsartan in Heart Failure: Emerging Clinical Data. Ther Clin Risk Manag 2020; 16:715-26.

17. McMurray JJV, Solomon SD, Inzucchi SE, Køber L, Kosiborod MN Martinez FA, et al. Dapagliflozin in Patients with Heart Failure and Reduced Ejection Fraction. N Engl J Med 2019;381(21):1995-2008.

18. Mesquita ET, Jorge AJL, Rabelo LM, Souza Júnior CV. Entendendo a Hospitalização em Pacientes com Insuficiência Cardíaca. Int J Cardiovasc Sci 2016;30(1):81-90.

19. Albuquerque DC, de Souza Neto JD, Bacal F, Rohde EPL, BernardezPereira S, Berwanger O, et al. I Registro Brasileiro de Insuficiência Cardíaca - Aspectos Clínicos, Qualidade Assistencial e Desfechos Hospitalares. Arq Bras Cardiol2015; 104(6):433-441.

20. Snipelisky D, Chaudhry SP, Stewart GC. The Many Faces of Heart Failure. Card Electrophysiol Clin2019; 11(1):11-20.

21. Carvalho AMF. Atualização em Insuficiência Cardíaca na Criança. Rev Saúde Criança Adolesc2011;3(1):81-92.

22. Zhang Y, Zhang J, Butler J, Yang X, Xie P, Guo D, et. al. Contemporary Epidemiology, Management, and Outcomes of Patients Hospitalized for Heart Failure in China: Results From the China Heart Failure (China-HF) Registry. J Card Fail 2017; 23(12):868-875.

23. Santos PM, Freire RB, Fernández AE, Sobrino J L B, Pérez CF, Somoza FJE, et. al. In-hospital Mortality and Readmissions for Heart Failure in Spain. A Study of Index Episodes and 30-Day and 1-year Cardiac Readmissions. RevEspCardiol(Engl Ed) 2019; 72(12)998-1004. 